\title{
Electron mobility in liquid and supercritical helium measured using corona discharges: a new semi-empirical model for cavity formation
}

\author{
F. Aitken, ${ }^{* a}$ Z.-L. Li, ${ }^{a}$ N. Bonifaci, $\dagger^{a}$ A. Denat ${ }^{a}$ and K. von Haeften ${ }^{b}$ \\ Received 4th June 2010, Accepted 15th September 2010 \\ DOI: $10.1039 / \mathbf{c 0 c p 0 0 7 8 6 b}$
}

Electron mobilities in supercritical and liquid helium were investigated as a function of the density. The mobilities were derived from $I(V)$ curves measured in a high-pressure cryogenic cell using a corona discharge in point-plane electrode geometry for charge generation. The presented data spans a wide pressure and temperature range due to the versatility of our experimental set-up. Where data from previous investigations is available for comparison, very good agreement is found. We present a semi-empirical model to calculate electron mobilities both in the liquid and supercritical phase. This model requires the electron-helium scattering length and thermodynamic state equations as the only input and circumvents any need to consider surface tension. Our semi-empirical model reproduces experimental data very well, in particular towards lower densities where transitions from localised to delocalised electron states were observed.

\section{Introduction}

Microscopic probes have always played an important role in the exploration of the unique properties of liquid helium (LHe). Examples of these are electrons. Electrons were used to identify the rotation of helium in single vortex lines ${ }^{1,2}$ and they were also used in early mobility measurements ${ }^{3}$ leading to the discovery of negative charge localisation in large voids. ${ }^{4}$ This localisation and the establishment of spherical 'cavities' around electrons at a critical density and temperature is the cause of a large drop in mobility of over more than four orders of magnitude. ${ }^{5}$ An electron confined in a cavity gives rise to quantised energy eigenstates and the existence of cavities was later nicely demonstrated when these states were probed by infrared spectroscopy. ${ }^{6,7}$

The formation of cavities has its origin in the predominantly repulsive interactions of ground state helium atoms. Their size results from the balance of forces of this repulsion and the surface energy. ${ }^{8,9}$ Electron cavity radii are typically on the order of $15 \AA$, but depend on the hydrostatic pressure. ${ }^{10}$ In dense helium gas, cavities exist as well. There, like in the normal liquid phase, the motion is governed by the laws of hydrodynamical (Stokes) flow, whereas in the superfluid state interactions with collective excitations (phonons, rotons and vortices) become dominant. ${ }^{11}$ In all of these flow regimes the radius of the spherical cavities determines the mobility. In the gas phase at lower densities, cavities are not supported. In this regime the mobility is governed by gas-kinetic type behaviour. With decreasing temperature and increasing density a point is reached where the mobility dramatically decreases. This point can be interpreted as the onset of cavity formation. Low temperatures assist the localisation process which is reflected by a shift in the mobility transition region to higher densities

${ }^{a}$ G2ELab-CNRS, 25 rue des Martyrs, 38042 Grenoble, France.

E-mail: frederic.aitken@grenoble.cnrs.fr

${ }^{b}$ Department of Physics \& Astronomy, University of Leicester,

Leicester, LE1 7RH, United Kingdom

$\dagger$ Also at Université Joseph Fourier, Grenoble, France. with increasing temperature. ${ }^{5}$ Cavity formation around electrons in LHe has been studied extensively using various methods ${ }^{12,13}$ including density function theory (DFT). ${ }^{14-17}$ DFT methods specially developed for LHe provide good agreement with first principles methods for small helium clusters, ${ }^{18}$ but have the disadvantage that they are restricted to zero Kelvin. Despite all these efforts, the precise prediction of the transition from gas-kinetic to hydrodynamic flow still remains a challenge for theory. Presently available models provide only limited accuracy in predicting electron mobilities, even when far from the transition zone. Furthermore, mobility data covering the transition density regime is rather sparse and makes it difficult to test existing theory.

We have therefore undertaken a series of measurements for liquid and supercritical helium using the method of corona discharges for charge generation. We derived mobilities from $I(V)$ curves measured in a point-plane electrode arrangement. ${ }^{19}$ Furthermore, we have developed a semi-empirical model to calculate mobilities and cavity radii. The precise determination of mobilities requires knowledge of the surface energy. Commonly, the surface tension of helium is employed, but this is problematic. ${ }^{20,21}$ The surface tension deviates for curved surfaces and is not defined for all hydrostatic pressures $(P)$ and temperatures $(T)$, especially in low density regions of supercritical gas. Adjusted values for the surface tension in $\mathrm{LHe}$ have been employed ${ }^{17}$ but do not resolve the problem. Fig. 1 visualises the difference between measured and predicted mobilities, using the popular 'bubble' model. The semiempirical thermodynamic model that we introduce in this paper circumvents the use of surface tension. It is based on the free-volume concept $\mathrm{t}^{2,23}$ and employs the electron scattering length and appropriate thermodynamic state equations as the only input.

Despite its semi-empirical nature our approach represents an important step towards a better understanding of the electron localisation process as it predicts mobilities and cavity radii with much greater precision than any other existing theory. Furthermore, it will be valuable for the microscopic 


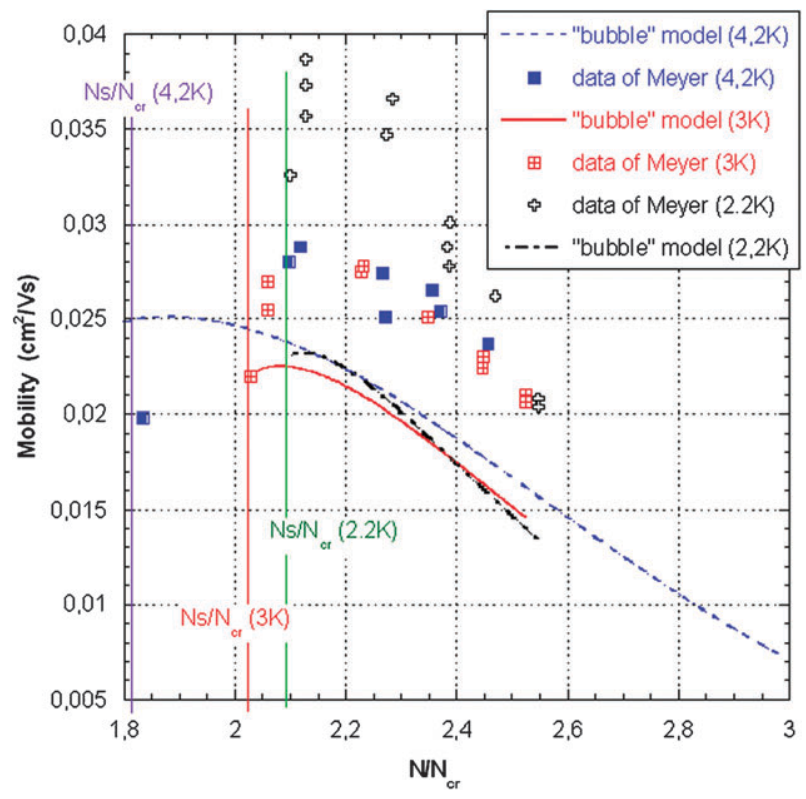

Fig. 1 Electron mobilities in LHe measured at 2.2, 3 and $4.2 \mathrm{~K}$ as a function of the normalised density $N / N_{\mathrm{cr}}$, where $N_{\mathrm{cr}}$ relates to the critical density. In this region in particular, the 'bubble'-model commonly used shows significant deviations from the experimental data, for instance, the maximum at $N / N_{\mathrm{cr}}=2.2$ cannot be reproduced. The three vertical lines at $N_{\mathrm{s}} / N_{\mathrm{cr}}$ refer to the ratio between the density at saturated vapour pressure $N_{\mathrm{s}}$ and the density at the critical point $N_{\mathrm{cr}}$ of $69.641 \mathrm{~kg} \mathrm{~m}^{-3}$.

probing of the properties of LHe or helium droplets. Cavities also exist around electronically excited helium atoms and helium excimers in Rydberg states. Although these species are neutral, their cavities are very similar to those around electrons. For neutral Rydberg species, the void is created by the repulsive interaction between the Rydberg-electron orbiting around the positive core with the surrounding ground state helium atoms. There are nevertheless differences in size because the Rydberg-electron is still attracted by the positive core. Neutral Rydberg-cavity systems are inaccessible to mobility measurements, but they provide a rich source of spectroscopic information as first demonstrated by Dennis and coworkers for high-energy electron-beam-excited bulk LHe, ${ }^{24,25}$ Femtosecond laser-excited $\mathrm{LHe}^{26,27}$ and for helium droplets photoexcited by synchrotron radiation. ${ }^{28-30}$ Recently, corona discharges have been employed to excite supercritical and bulk $\mathrm{LHe}$ and a rich fluorescence spectrum similar to that when using electron beams and synchrotron radiation was observed..$^{31,32}$ Rydberg-cavities are important as spectroscopic probes of microscopic states of helium. The concept for deriving the size of electron cavities is transferable to Rydberg cavities and will be relevant for future investigations of LHe.

The electron mobilities presented in this paper were measured in supercritical gas at 6,7 and $10 \mathrm{~K}$ as well as in LHe at $4.2 \mathrm{~K}$. The advantage of using point-plane electrode geometry in a corona discharge is that it naturally generates electrons and that it provides for the establishment of very high pressures so that a wide density range can be covered. Where data is available in the literature, we find a good match with our results. Our semi-emipirical model fits the measured electron mobilities better than any other available model. The improvement is particularly evident for supercritical helium.

\section{Experiment}

Mobility measurements were carried out in a high-pressure cell that was attached to a bath cryostat using a corona discharge to generate the charge carriers. Details of the set-up were published in previous publications ${ }^{31-33}$ and here we give only a brief description. Helium gas of purity level 99.9999\% was passed through a series of liquid nitrogen-cooled cold traps and activated charcoal filters and introduced into the high-pressure cell. The corona discharge was established in point-plane geometry. For this geometry, previous workers have found that the current is unipolar space charge-limited and concentrated along the tip axis, ${ }^{34,35}$ both for gases and liquids. In liquids, the current depends quadratically on the voltage:

$$
I=C_{t} \mu \varepsilon \frac{\left(V-V_{0}\right)^{2}}{d}
$$

where $I$ represents the current, $d$ the distance between tip and plane, $C_{\mathrm{t}}$ is a constant, $\mu$ the mobility, $\varepsilon$ the dielectric constant $\left(\varepsilon_{0} \varepsilon_{\mathrm{r}}\right)$ and $V$ and $V_{0}$ the voltage and the threshold voltage. ${ }^{35}$ The mobility can be obtained from a linear fit of the square root of the current plotted against the voltage. In this plot the straight, asymtotic part of the $\sqrt{I(V)}$ graph is identical to the mobility. $C_{t}$ in eqn (1) is derived by comparison with experiments using other methods such as the time of flight for the derivation of the mobility. Taking $C_{t}=2$ leads to a good agreement with experiment ${ }^{36}$ as well as with theory. ${ }^{37}$

\section{Results}

$I(V)$ curves were recorded for 4.2, 6, 7 and $10 \mathrm{~K}$ as a function of the hydrostatic pressure and mobilities were derived using eqn (1) according to the above procedure. Fig. 2 shows the electron mobilities at $4.2 \mathrm{~K}$ alongside the mobilities as reported in the literature. ${ }^{38,39}$ Also, the mobilities have been calculated using the common bubble method, as well as our model.

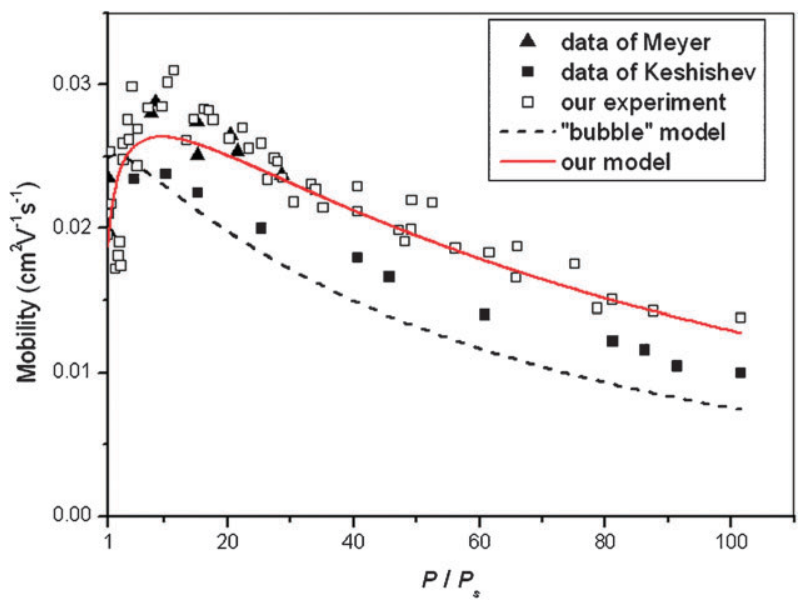

Fig. 2 Pressure dependence of electron mobilities measured at $4.2 \mathrm{~K}$ by Meyer et al. ${ }^{38}$ Keshishev et al. ${ }^{39}$ and in this work. The pressures are normalised to the saturated vapour pressure $P_{\mathrm{s}}=0.9847$ bar. 


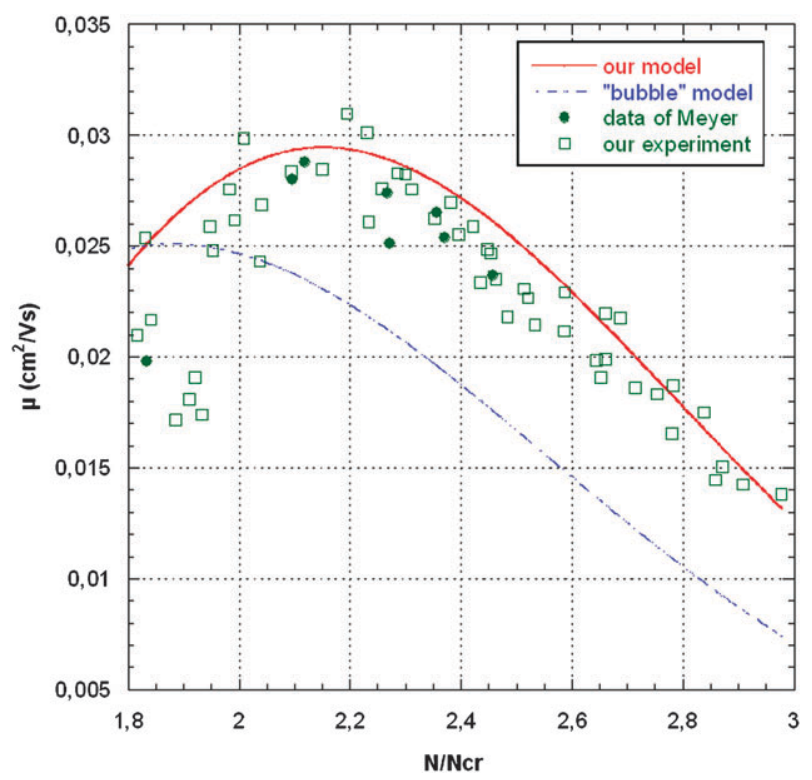

Fig. 3 Density dependence of electron mobilities measured in $\mathrm{LHe}$ at $4.2 \mathrm{~K}$ by Meyer et al. ${ }^{38}$ and by us (this work). The densities are normalised to the critical density of helium of $0.0694 \mathrm{~g} \mathrm{~cm}^{-3}$. The dashed line refers to calculated electron mobilities using the established 'bubble'-model and the solid line shows the results using our model.

The data shows that the mobilities increase with pressure, pass through a maximum and decrease again. Our data covers particularly the low pressure region (0.1-1 MPa) since electron mobilities in this pressure range have only been sparsely reported in the literature. Fig. 3 shows the mobilities in $\mathrm{LHe}$ as a function of the normalised density.

We also investigated the effect of the tip size by using two different tips with a radius of 0.45 and $2.5 \mu \mathrm{m}$, respectively, to ascertain that the way we derive the mobilities from the $I(V)$ curves works. No difference was observed.

\section{Discussion}

In helium gas at low density the electron is free and its mobility $\mu_{0}$ is governed by the laws of gas-kinetics:

$$
\mu_{0}=\frac{4 e}{3 N q\left(2 \pi m_{e} k_{b} T\right)^{1 / 2}}
$$

where e and $\mathrm{m}$ are the electron charge and mass, respectively, $N$ the number density, $q$ the momentum transfer cross-section (constant for helium, $q=5 \times 10^{-20} \mathrm{~m}^{2}$ ) and $k_{\mathrm{B}}$ the Boltzmann constant.

At higher gas densities an electron can interact with several atoms simultaneously ${ }^{40}$ and consequently the mobility $\mu_{0}$ that was derived in eqn (2) on the assumption of single atom-electron interactions does not fully match experimental data. These deviations can be corrected using the following expression:

$$
\mu=\mu_{0}\left(1-\sqrt{\pi} \mu_{e} N q\right)
$$

where $\mu$ refers to the corrected mobility and $\lambda_{e} N q$ to the ratio between the electron de Broglie wavelength $\lambda_{\mathrm{e}}$ and the classical mean free path length $1 / N q$. Eqn (3) is only accurate for small $\lambda_{e}$ (fast electrons) and as it takes only first order density corrections into account it is only valid for moderate corrections $\mu / \mu_{0}>0.3$.

The mobilities $\mu$ calculated in this way agree well with experimental data up to densities $N / N_{\text {cr }} \cong 0.15$, where $N_{\text {cr }}$ refers to the critical density. At higher relative densities $N / N_{\text {cr }}$ a transition from a quasi-free to a localised state takes place which is observed through an abrupt decrease of the electron mobility from $100 \mathrm{~cm}^{2} \mathrm{~V} \mathrm{~s}^{-1}$ to $0.1 \mathrm{~cm}^{2} \mathrm{~V} \mathrm{~s}^{-1}$. In this region, below $0.1 \mathrm{~cm}^{2} \mathrm{~V} \mathrm{~s}^{-1}$, the Knudsen number becomes lower than 1 and therefore a hydrodynamical analysis, similar to the one proposed for $\mathrm{LHe}$, can be used. Measurements of the mobility in the transition region are afflicted with a very large error because the changes are so rapid.

The physical process of electron-localisation can be explained by the minimisation of the large interaction energy between the electron and helium atoms leading to the self-trapping of free electrons in a void. Schmidt et al. described the trapping to proceed in multiple steps, the first step being the occasional localisation of the excess electron in an unstable density fluctuation of the liquid, and the second being stabilisation via the formation of a larger cavity. ${ }^{41}$ The driving force in this process is the Pauli repulsion between the excess electron and the surrounding helium atoms, with both of their electrons residing in the $1 \mathrm{~s}^{2}$ state, due to the Pauli principle. The electron pushes the helium atoms away thereby acting against the restoring force of the surface tension $\sigma(P, T)$ until an equilibrium cavity radius $R$ is reached. In the first approximation, it is the increased geometric cross-section of this cavity that gives rise to the comparatively low mobilities of electrons in supercritical gas and LHe. From hydrodynamics we know that for small velocities the viscous force $F_{f}$ exerted on a sphere follows Stokes law. Hence, the relation between mobility $\mu$ and cavity radius $R$ is as follows:

$$
\begin{aligned}
e E-F_{f} & =0 \rightarrow e E-4 \pi R \eta \mathrm{v}=0 \\
e E & =4 \pi R \eta \mu E \rightarrow \mu=\frac{e}{4 \pi R \eta}
\end{aligned}
$$

Here, $E$ is the electric field strength and $\eta(P, T)$ the dynamic viscosity of the fluid. We note that for the 'bubble model' a coefficient of $0.8 \times 4 \pi$ is commonly used ${ }^{12,42,43}$ because the coefficient of $4 \pi$ in the Stokes equation applies to bubbles whereas the more familiar $6 \pi$ is due to drops. ${ }^{44,45}$ It is reasonable to assume a spherical shape of the 'cavity' since the interaction between electron and helium atoms is isotropic and therefore the radius $R$ of a cavity can be derived from mobility data.

It is also possible to calculate the cavity radius from the equilibrium of forces acting on the cavity. The most frequently adopted approach calculates the equilibrium radius $R$ by minimising the electron excess free energy with respect to the radius. The free energy is represented by the zero-point energy of an electron in a square-well-potential, the surface energy represented by the product of surface tension $\sigma(P, T)$ and cavity surface and the volume displacement work of the liquid. $^{43}$

$$
E_{\text {cavity }}=\frac{h^{2}}{8 m R^{2}}+4 \pi R^{2} \sigma+\frac{4}{3} \pi R^{3} P
$$


Where $h$ is Planck's constant, $m$ the electron mass, $\sigma$ the surface tension and $P$ the hydrostatic pressure.

The agreement of the mobilities calculated in this way with experimental results is fair for high pressures $(P>1 \mathrm{MPa})$ but with lower and lower pressures, deviations become apparent. Particularly, the position of the maximum observed in Fig. 1 for low pressures or densities, respectively, cannot be reproduced when using eqn (5). Furthermore, this model cannot reproduce the variation in mobility as a function of pressure at different temperatures. The reason for these deviations lies in the more complicated structure than that of a simple "empty bubble" with a sharp interface. It is very reasonable to assume that the thickness of the cavity-liquid interface is of similar order to the liquid-vapor interface of LHe, which is $7 \AA, 46$ and which is not small compared to the cavity radius. Therefore, the use of the concept of surface tension is not applicable here. A better approach is to transform the surface energy into a volume energy similar to that of the work against hydrostatic pressure $P V$. A second reason for the deviations is that the surface tension $\sigma(P, T)$ is used for $P$ and $T$ values where it is not defined. The surface tension is only defined at gas-liquid interface systems that are governed by the well-known saturated vapour-pressure relationship between $P$ and $T$, but this relationship does not necessarily correspond to the conditions at the interface between cavity and liquid.

In this section we demonstrate how mobilities can be calculated more accurately. Essentially, we develop the thermodynamic equation of state for excess electrons in helium in the limit of low concentrations using the free-volume concept. The mobilities calculated in this way show the pressure and temperature dependence without the limitation imposed by using the surface tension. In the past, the free-volume concept was successfully used to predict the properties of liquids and solids, such as the ionic conductivity. ${ }^{22,23}$ Our approach is more generic than the cavity model as it predicts the volume of cavities formed by the electrons regardless of its specific structure. The ingredients to calculate the size of the void that we use are the scattering length $a$ for electrons in helium to represent the Pauli repulsion and an appropriate thermodynamic state equation to represent attraction, i.e. the restoring forces similar to that of surface tension. The advantage of our model is its applicability to thermodynamic states in a much wider range, in particular to states with low density. We will see that much better agreement with experimental data can be achieved.

Our idea is based on the proportionality between the volume of the object formed by excess electrons $V_{\mathrm{e}}$ and the 'free-volume' $(V-b)$ of the object where the term 'free volume' refers to the notation of the van der Waals state equation. The proportionality holds exact if the total volume is not changed too much when foreign objects are introduced. For excess electrons this condition is clearly fulfilled since their quantity is negligible compared to the total number of helium atoms in the discharge cell. We replace $(V-b)$ by $V_{f}$ and write for the volume $V_{e}$ of an electron cavity with arbitrary shape:

$$
V_{e}=C \frac{V_{f}}{N}
$$

where $C$ is a constant of proportionality which we obtain here by fitting to the experimental data at $3 \mathrm{~K}$ (any other temperature can be chosen as well) and $N$ the number of helium particles that are displaced by the foreign object. In this notation a spherical cavity formed by the electron has the volume $V_{e}=\frac{4}{3} \pi R^{3}$ and moves within the Stokes flow regime giving rise to a mobility $\mu$ related to $R$.

The free volume of LHe is a function of two independent thermodynamic parameters: $P$ and $T$. The relation between these quantities can be expressed through the classical van der Waals equation of state:

$$
\left(P+\Pi_{\mathrm{LHe}}\right) \frac{V_{f}}{N}=k_{\mathrm{B}} T
$$

where $\Pi_{\mathrm{LHe}}$ is the internal pressure. The internal pressure represents the attractive forces in the system (i.e. long range van der Waals interaction) and generally depends on the temperature $T$ and the particle density $\rho$.

Our aim is now to develop an expression for $\Pi_{\mathrm{LHe}}$ that accounts best for the physical properties of our system. We start with the following generic equation to describe the dependence on local variations of the density:

$$
\Pi_{\mathrm{LHe}}=f_{1}(T) \rho^{2}+\text { terms }
$$

The extra terms in this equation account for the 'local' internal pressure close to the foreign object where the attractive forces are non-isotropic. In our model we have chosen the following expression:

$$
\Pi_{L H e}=\frac{\alpha \rho^{2}+\beta\left(\exp \gamma \frac{\rho}{\rho_{s}}-\exp \gamma\right)}{T^{2}}
$$

where $\rho / \rho_{\mathrm{s}}$ is the ratio between the density and the density of the liquid for saturated vapor pressure at a given temperature $T$ and $\alpha=0.007, \beta=4, \gamma=4.9$. We note that when $\rho$ approaches $\rho_{\mathrm{s}}$ the second term vanishes and the equation for the internal pressure assumes the van der Waals form.

For a supercritical gas eqn (9) has to be modified because the only reference is the critical density. Therefore, it is reasonable to assume a similarity between the parameter $\Pi_{\text {SupHe }}$ referring to the state equation in supercritical helium and $\Pi_{\mathrm{LHe}}$ with the supercritical density $\rho_{\mathrm{s}}$ replaced by the critical density $\rho_{\mathrm{c}}$ and without the factor $\mathrm{e}^{\gamma}$. The state equation for $\Pi_{\text {SupHe }}$ assumes then the following form:

$$
\Pi_{\mathrm{SupHe}}=\frac{\alpha \rho^{2}+\beta \exp \frac{\rho}{\rho_{c}}}{T^{2}}
$$

with the parameter $\alpha=0.007, \beta=4$ of the liquid.

To derive the radius $R$ of the cavity we assume proportionality with the scattering length $a$ and exploit the inverse proportionality with the free volume $V_{f}$. Hence, we find the following empirical relation for the $a$ and $V_{f}$ dependence of the cavity radius $R$ :

$$
R=a \frac{\left(V_{\text {ref }} / V_{f}\right)^{3}}{1+0.09\left(V_{\text {ref }} / V_{f}\right)^{3} \exp \left(\frac{-1.7}{V_{\text {ref }} / V_{f}}\right)}
$$

$V_{f}$ can be expressed through the state equation $V_{\text {ref }} / V_{f}=$ $4.5 \frac{p+\Pi}{T}$ and it is then possible to calculate the cavity radius as a function of $P$ and $T$ by fitting the parameters in our empirical 
relation to the measured values of the radius derived from the measured mobilites through the Stokes equation.

$$
R=a \frac{\left(4.5 \frac{P+\Pi}{T}\right)^{3}}{1+0.09\left(4.5 \frac{P+\Pi}{T}\right)^{3} \exp \left(\frac{-1.7}{\left(4.5 \frac{P+\Pi}{T}\right)}\right)}
$$

In this equation expressions for the internal pressure $\Pi$ have to be entered according to eqn (9) or (10) depending on whether liquid or supercritical helium is considered. All pressures have to be entered in units of bar; the scattering length $a$ is $0.062 \mathrm{~nm}$; all constants are real constants and independent of the temperature. The behavior at the limits $\rho \rightarrow 0$ is $R \rightarrow \sim a$ and $\rho \rightarrow \infty$ is $R \rightarrow \sim 11 \mathrm{~nm}$.

In the regime of supercritical gas densities where a hydrodynamical approach can be used (Knudsen number $<1$ ) the agreement with experimental data is very good (Fig. 3). Fig. 4 shows the measured and calculated electron mobilities for liquid and supercritical helium over a wide range of densities. The dashed line refers to the common bubble model. It can be seen that our model produces very good fits in both the liquid and the supercritical regime.

It is difficult to assign the true origin of the rapid change in electron mobility in the transition region. Firstly, this region is characterised by a cross-over from hydrodynamic to molecular flow, but this transition should be a gradual change, much less rapid than the observed change in mobility. Secondly, in the framework of hydrodynamic flow there are two possibilities to explain the increase of the electron mobility. On the one hand, for decreasing density $\rho \rightarrow 0$ the viscosity also decreases

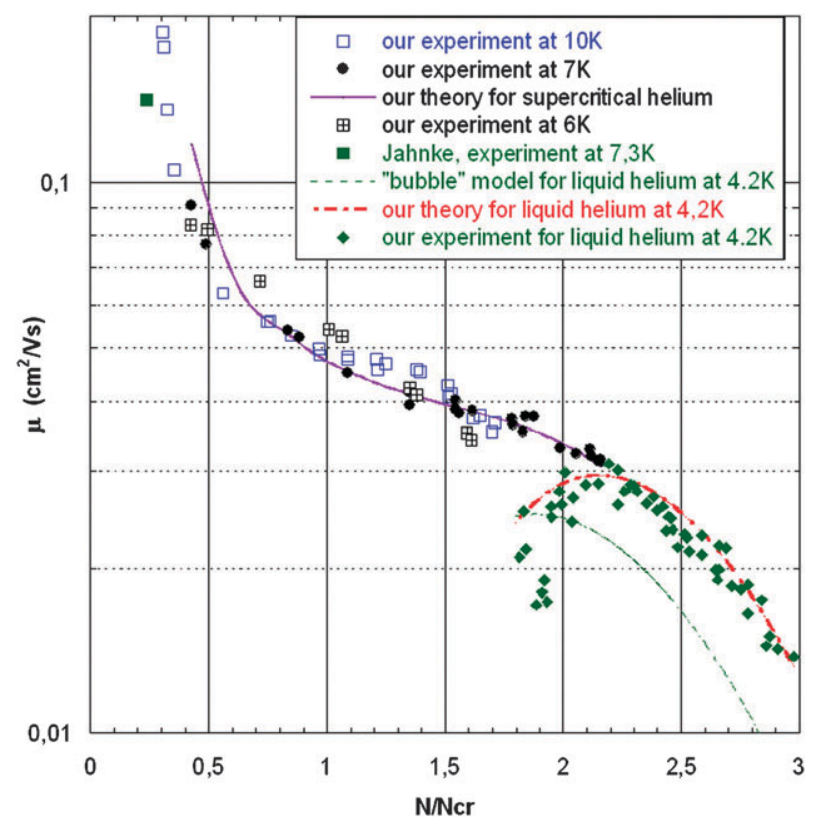

Fig. 4 Density dependence of electron mobilities at 4.2, 6, 7 and $10 \mathrm{~K}$. The figure shows measured data by Jahnke et al., ${ }^{47}$ this work and calculated data using the common bubble model (dashed green line) and our approach (dashed-dotted red and violet solid lines). The violet solid line refers to our calculation for supercritical helium. Again, the densities are normalised to the critical density of helium of $0.0694 \mathrm{~g} \mathrm{~cm}^{-3}$. $\eta \rightarrow 0$, but this decrease is relatively slow. On the other hand, it is also possible that the cavity radius $R$ increases. The experimental values show that this decrease in $R$ must be more rapid than that of $\eta$. The fact that the cavity shrinks is in contradiction with the 'bubble' model commonly used in the literature. In this model the force acting against the electron repulsion is the surface tension which is expected to decrease with decreasing density. Hence, one would expect the cavity to increase in size which clearly contradicts the experimental data. Up to now there is no satisfying explanation of why the mobility changes are so dramatic and why they take place particularly at $N / N_{\text {cr }} \cong 0.15$.

\section{Conclusions}

In summary, we have presented new data on electron mobilities in supercritical and liquid helium. The new data allowed us to elaborate the density dependence in a region which is only sparsely covered in the existing literature. To cover the widest possible density range we generated free electrons in a corona discharge using point-plane electrode geometry. This relatively simple and versatile set-up allowed us to establish pressures of up to 100 bar and temperatures down to $4 \mathrm{~K}$. The mobilities were also simulated. We developed a new model for the hydrodynamical flow regime that derives the electron mobilities from the electron scattering length in helium $a$ representing repulsion and an appropriate thermodynamic state equation where the internal pressure $\Pi$ accounts for the attraction. This approach circumvents the use of the surface tension as commonly used in the 'bubble' model. The common 'bubble' model is an easy-to-understand concept, but is has some shortcomings: (i) it predicts cavity growth for low-densities which is contradictory to experimental data and (ii) it employs surface tension values to thermodynamic states where these are not defined. Our model overcomes these shortcomings as it predicts the density-dependence of the electron mobility correctly. Also, the accuracy is unsurpassed by any other presently available model. Our semi-empirical model makes use of parameters that are adjusted to experimental data. This adjustment is made only once and the resulting equation to calculate electron mobilities or cavity sizes is universally applicable for all thermodynamic states within the molecular flow region. In addition to its practical use, our model may be useful to shed more light on the microscopic properties of cavities in liquid and supercritical helium. For instance, if a microscopic theory of Stokes flow existed, more information on the cavity-liquid interface could be obtained because our model makes no assumption of the actual shape of the cavities. It just assigns a hypothetical sphere of displaced helium atoms to the free volume.

\section{Acknowledgements}

F. Aitken and K. von Haeften acknowledge funding through the Alliance programme by the British Council. K. von Haeften also acknowledges funding through a Leverhulme Trust Research Grant. 


\section{References}

1 R. Packard and T. Sanders Jr, Phys. Rev. A: At., Mol., Opt. Phys., 1972, 6, 799-807.

2 G. Williams and R. Packard, Phys. Rev. Lett., 1974, 33, 280-283.

3 J. Levine and T. M. Sanders, Phys. Rev. Lett., 1962, 8, 159-161.

4 J. Levine and T. Sanders, Phys. Rev., 1967, 154, 138-149.

5 A. Borghesani and M. Santini, Phys. Rev. E: Stat., Nonlinear, Soft Matter Phys., 2002, 65, 56403.

6 C. Grimes and G. Adams, Phys. Rev. B: Condens. Matter, 1990, 41, 6366-6371.

7 A. Golov, Z. Phys. B: Condens. Matter, 1995, 98, 363-366.

8 J. Jortner, N. R. Kestner, S. A. Rice and M. H. Cohen, J. Chem. Phys., 1965, 43, 2614-2625.

9 B. Springett, J. Jortner and M. Cohen, J. Chem. Phys., 1968, 48, 2720.

10 W. Fowler and D. Dexter, Phys. Rev., 1968, 176, 337-343.

11 A. Borghesani, Ions and Electrons in Liquid Helium, Oxford University Press, USA, 2007.

12 J. Classen, C. Su, M. Mohazzab and H. Maris, Phys. Rev. B: Condens. Matter Mater. Phys., 1998, 57, 3000-3010.

13 M. Barranco, M. Guilleumas, M. Pi and D. M. Jezek, Approaches to Quantum Liquids in Confined Geometries, ed. E. Krotscheck and J. Navarro, World Scientific, Singapore, 2002, p. 319.

14 Q. Xiong and H. Maris, J. Low Temp. Phys., 1989, 77, 347-369.

15 F. Ancilotto and F. Toigo, Phys. Rev. B: Condens. Matter, 1994, 50, 12820.

16 J. Eloranta and V. Apkarian, J. Chem. Phys., 2002, 117, 10139-10150.

17 M. Pi, M. Barranco, R. Mayol and V. Grau, J. Low Temp. Phys., 2005, 139, 397-417.

18 F. Dalfovo, Z. Phys. D, At., Mol. Clusters, 1994, 29, 61-66.

19 X. Li, Y. Tang, L. Lin and J. Li, Microporous Mesoporous Mater., 2008, 111, 591-595.

20 J. Treiner and H. Krivine, Ann. Phys., 1986, 170, 406- 453.

21 Y. Rocard, Thermodynamique, Masson, 1952.

22 A. Doolittle, J. Appl. Phys., 1951, 22, 1471.

23 T. Miyamoto and K. Shibayama, J. Appl. Phys., 1973, 44, 5372.

24 W. S. Dennis, J. E. Durbin, W. A. Fitzsimmons, O. Heybey and G. K. Walters, Phys. Rev. Lett., 1969, 23, 1083-1086.

25 J. Keto, F. Soley, M. Stockton and W. Fitzsimmons, Phys. Rev. A: At., Mol., Opt. Phys., 1974, 10, 872-886.
26 A. Benderskii, R. Zadoyan, N. Schwentner and V. Apkarian, J. Chem. Phys., 1999, 110, 1542.

27 A. Benderskii, J. Eloranta, R. Zadoyan and V. Apkarian, J. Chem. Phys., 2002, 117, 1201.

$28 \mathrm{~K}$. von Haeften, A. R. B. de Castro, M. Joppien, L. Moussavizadeh, R. von Pietrowski and T. Möller, Phys. Rev. Lett., 1997, 78, 4371.

29 K. von Haeften, T. Laarmann, H. Wabnitz and T. Möller, Phys. Rev. Lett., 2002, 88, 233401.

30 K. von Haeften, T. Laarmann, H. Wabnitz, T. Möller and J. Phys. B, J. Phys. B: At., Mol. Opt. Phys., 2005, 38, S373-S386.

31 Z. Li, N. Bonifaci, F. Aitken, A. Denat, K. von Haeften, V. Atrazhev and V. A. Shakhathov, Eur. Phys. J. Appl. Phys., 2009, 52, 11.

32 Z. Li, N. Bonifaci, F. Aitken, A. Denat, K. von Haeften, V. Atrazhev and V. A. Shakhathov, IEEE Trans. Dielectr. Electr. Insul., 2009, 16, 742-750.

33 Z. Li, N. Bonifaci, A. Denat and V. Atrazhev, Dielectrics and Electrical Insulation, IEEE Transactions on [see also Electrical Insulation, IEEE Transactions on], 2006, 13, 624-631.

34 B. Halpern and R. Gomer, J. Chem. Phys., 1969, 51, 1031.

35 P. Sibillot and R. Coelho, J. Phys., 1974, 35, 141-148.

36 N. Bonifaci, A. Denat and B. Malraison, IEEE Trans. Ind. Appl., 2001, 37, 1634-1640.

37 R. Sigmond, J. Appl. Phys., 1982, 53, 891.

38 L. Meyer, H. Davis, S. Rice and R. Donnelly, Phys. Rev., 1962, 126, 1927-1934.

39 K. Keshishev, Y. Kovdrya, L. Mezhov-Deglin and A. Shalnikov, Sov. Phys. JETP, 1969, 56, 94.

40 V. Atrazhev, J. Phys. D: Appl. Phys., 1984, 17, 889-902.

41 W. F. Schmidt, E. Illenberger, A. Khrapak, Y. Sakai and K. Yoshino, IEEE Trans. Dielectr. Electr. Insul., 2003, 10, $1012-1021$.

42 H. Maris, J. Low Temp. Phys., 2003, 132, 77-95.

43 H. Maris, J. Phys. Soc. Jpn., 2008, 77, 111008.

44 G. Batchelor, An Introduction to Fluid Dynamics, Cambridge University Press, 1970.

45 A. Khrapak, W. Schmidt and E. Illenberger, Electronic Excitations in Liquefied Rare Gases, 2005, 239-273.

46 K. Penanen, M. Fukuto, R. Heilmann, I. Silvera and P. Pershan, Phys. Rev. B: Condens. Matter Mater. Phys., 2000, 62, 9621-9640.

47 J. Jahnke, M. Silver and J. Hernandez, Phys. Rev. B: Solid State, 1975, 12, 3420-3427. 